\title{
Aromatase inhibitors in the treatment of breast cancer in post-menopausal female patients: an update
}

This article was published in the following Dove Press journal:

Breast Cancer:Targets and Therapy

3 October 2011

Number of times this article has been viewed

\author{
Reva Schneider' \\ Ayman Barakat' \\ John Pippen ${ }^{1,2,3}$ \\ Cynthia Osborne $e^{1,2,3}$ \\ 'Medical Oncology, Baylor-Sammons \\ Cancer Center, ${ }^{2}$ Texas Oncology PA \\ ${ }^{3}$ US Oncology, Dallas, TX, USA
}

\begin{abstract}
Estrogen and its metabolites play a significant role in the proliferation of hormone receptor-positive breast cancer. In postmenopausal women, aromatase inhibitors can significantly reduce estrogen levels by blocking enzyme-mediated estrogen synthesis within tissues. Third-generation aromatase inhibitors have now surpassed tamoxifen as first-line therapy for postmenopausal women with metastatic, hormone receptor-positive, breast cancer, showing improved response rates and time to progression. Aromatase inhibitors have shown incremental improvements in disease-free survival, lower local recurrence rates, lower metastatic recurrence rates, and a lower incidence of contralateral breast cancer over tamoxifen when used in the adjuvant setting. Aromatase inhibitors are recommended to be used as adjuvant therapy within the first 5 years of hormonal therapy and may be used either upfront for 5 years or sequenced with tamoxifen. No superiority of one aromatase inhibitor over another has yet been shown. The side effect profiles of aromatase inhibitors have some key differences compared with tamoxifen. These differences may influence treatment choices as well as impact compliance.
\end{abstract}

Keywords: aromatase inhibitor, breast cancer, postmenopausal, hormonal therapy

\section{Introduction}

Breast cancer is the most common cancer in the US among women, is the second most common cause of cancer death in women, and the main cause of death in women aged 40-59 years. ${ }^{1}$ In the US in 2010 there were an estimated 207,090 new cases of breast cancer and 39,840 deaths, as reported by the National Cancer Institute.

There are significant data implicating estrogen and its metabolites in the development of breast cancer. Estrogen binding to its receptor results in proliferation of estrogen-sensitive epithelial tissues. With rapid proliferation, there is the potential for genetic errors and consequent predisposition to malignant cellular transformation. ${ }^{2}$ Alternatively, estrogen metabolites may have intrinsic genotoxic effects and cause DNA damage and cellular transformation. . $^{3,4}$

The use of hormonal treatments has been evolving since 1895 when George Thomas Beatson performed a bilateral oophorectomy on a woman with an extensive soft tissue recurrence of breast cancer resulting in a transient complete clinical remission. ${ }^{5}$ Now the pharmacological manipulation of estrogen via competitive blockade of the receptor or reduction of endogenous estrogen has become a mainstay of therapy for hormone receptor-positive breast cancer.

Tamoxifen, a selective estrogen receptor modulator, has been the gold standard of care for hormone-responsive breast cancer in both premenopausal and postmenopausal women. Tamoxifen effectively competes with estrogen for the estrogen 
receptor, disrupting downstream estrogen-driven signaling. A meta-analysis conducted by The Early Breast Cancer Trialists' Collaborative Group has shown that 5 years of adjuvant tamoxifen therapy significantly reduces the annual recurrence rate by almost half and results in a $31 \%$ proportional reduction in mortality. ${ }^{6}$ The effects were seen immediately and were persistent, with a mortality benefit ongoing at 15 years.

Estrogen-mediated signaling may also be interrupted via reduction of the estrogen substrate. Pharmacological means of reducing estrogen via aromatase inhibitors has become a relatively new therapeutic option in the breast cancer arena. Multiple, large, randomized trials have now been conducted utilizing third-generation aromatase inhibitors in both the advanced and early settings. This review discusses these agents, including their mechanism of action, pharmacological differences, side effects, and current usage in advanced and adjuvant settings.

The use of aromatase inhibitors for prevention and treatment of breast cancer is limited to patients without functional ovaries. In premenopausal women, aromatase inhibition inadequately suppresses estrogen production. ${ }^{7}$ In fact, these drugs have been used successfully to induce ovulation in cases of anovulatory infertility. ${ }^{8}$ Aromatase inhibitors should not be used alone to treat premenopausal or perimenopausal women, and all discussions here pertain to postmenopausal women only.

\section{Mechanisms of action and pharmacological properties}

Aromatase is a cytochrome P450 enzyme complex which is encoded by the CYP19 gene located on chromosome 15q21.2. It is expressed in the ovaries as well as several extragonadal tissues, including subcutaneous fat, brain, liver, bones, vascular endothelium, and mesenchymal cells of adipose tissue in the breast. ${ }^{9}$ The action of aromatase converts androgenic precursors (androstenedione and testosterone) to estrogen products (estrone and estradiol).

In premenopausal women, the vast majority of estrogen is synthesized in the granulosa cells of the ovaries under the influence of the hypothalamic-pituitary-ovarian axis. Estrogenic effects in premenopausal women are systemic and under endocrine control. In contrast, in postmenopausal women, estrogen synthesis takes place via local tissue synthesis that occurs in all of the previously mentioned sites and activity is primarily local. Thus, systemic measurement of estrogen in postmenopausal women is not a true reflection of local tissue estrogen concentrations. Tissue concentrations may in reality be many times higher than that measured systemically. ${ }^{10}$ In addition, it has been demonstrated that CYP19 expression, and thus aromatase activity, can be greatly increased in malignant as compared with normal breast tissue. This results in heightened intratumoral concentrations of estrogen. ${ }^{11-13}$ The potential for malignant-driven estrogen production and dependence makes inhibition of aromatase an appealing target for breast cancer therapy.

Aromatase inhibitors are classified as steroidal (type I) and nonsteroidal (type II, Figure 1). The steroidal compound drugs (formestane and exemestane) are analogs of the natural substrates, testosterone and androstenedione. ${ }^{14}$ They bind competitively to the substrate-binding site of the enzyme, forming tight, irreversible covalent bonds that result in permanent enzyme inactivation (suicide inhibitors). Reactivation of aromatase activity is dependent on continued synthesis of the enzyme. However, type II nonsteroidal aromatase inhibitors reversibly interact with the heme moiety of the cytochrome P450 subunit of P450 aromatase and inhibit steroidal aromatization. Therefore, sustained inhibition of aromatase depends on continuous drug presence. ${ }^{14}$

Aminoglutethimide is a first-generation nonsteroidal aromatase inhibitor and was the first aromatase inhibitor used clinically in the treatment of advanced breast cancer. Its mechanism of action is not selective for aromatase alone, but it more globally inhibits steroid synthesis, mimicking a surgical adrenalectomy. Usage requires hydrocortisone supplementation, and its side effect profile has limited its clinical utility. It is no longer widely available in the US. The second-generation steroidal inhibitor, formestane, requires intramuscular administration and has never been available in the US, although it has been utilized in other countries. However, it has been largely supplanted by the three orally available third-generation aromatase inhibitors, ie, anastrozole and letrozole, both of which are nonsteroidal inhibitors, and the irreversible steroidal inhibitor, exemestane.

\section{Clinical data on aromatase inhibitors Advanced disease}

A number of second-line metastatic breast cancer trials have been conducted comparing the progestational agent, megesterol acetate, and a third-generation aromatase inhibitor following tamoxifen failure. At a minimum, these selective aromatase inhibitors demonstrated equivalent if not superior efficacy to megesterol acetate in patients with tamoxifenresistant advanced metastatic breast cancer as a second-line therapy, ${ }^{15-17}$ thus paving the way for direct head-to-head 
Androgen substrate

Androstenedione<smiles>CC12CCC3C(CCC4=CC(=O)CCC43C)C1CCC2=O</smiles>

Testosterone

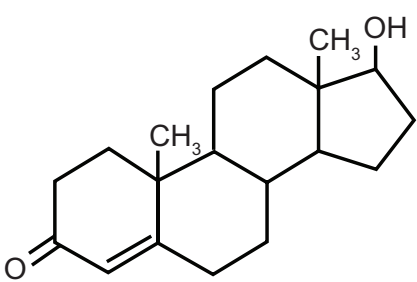

Steroidal inactivators

(Type I; irreversible)

Formestane

2nd generation<smiles>CC12CCC3C(CCC4=C(O)C(=O)CCC43C)C1CCC2=O</smiles>

Exemestane

3rd generation

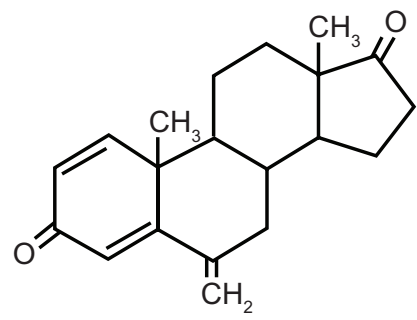

Non-steroidal inhibitors

(Type II; reversible)

Anastrozole

3rd generation<smiles>CC(C)(C)c1cc(Cn2cncn2)cc(C(C)(C)C#N)c1</smiles>

Letrozole

3rd generation

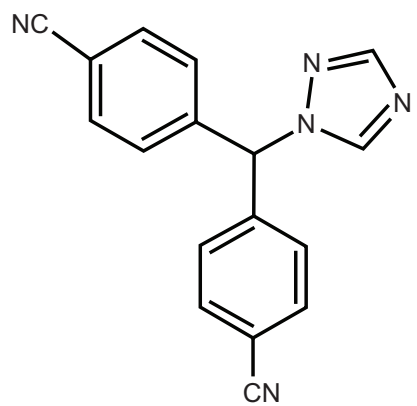

Figure I Structures of androgen substrates and aromatase inhibitors.

comparisons with the established first-line hormonal agent, tamoxifen (Table 1).

Anastrozole was the first of the selective third-generation aromatase inhibitors to be compared directly with tamoxifen. Two large randomized Phase III trials, ie, the North American Trial (conducted in the US and Canada), and TARGET (Tamoxifen or Arimidex Randomized Group Efficacy and Tolerability, conducted in Europe, Australia, New Zealand, South Africa, and South America) were conducted to compare tamoxifen and anastrozole as a first-line therapy for metastatic breast cancer with respect to time to disease progression, objective response rate, and tolerability. ${ }^{18,19}$ These trials were prospectively designed for a combined analysis.

In TARGET, anastrozole demonstrated equivalence in comparison with tamoxifen in terms of median time to disease progression ( 8.2 vs 8.3 months), objective response rate (32.9\% vs 32.6\%), and clinical benefit rate, defined as objective response rate and stabilization of disease for $\geq 24$ weeks (56.2\% vs $55.5 \%) .{ }^{19}$ In contrast, in the US trial there was a statistically insignificant improvement in objective response rate in favor of anastrozole ( $21 \%$ vs $17 \%$ for tamoxifen). However, a significant improvement in median time to disease progression was shown for treatment with anastrozole of 11.1 months in comparison with 5.6 months for tamoxifen treatment $(P=0.005)$. In addition, the clinical benefit rate was significantly higher with anastrozole $(59.1 \%)$ compared with tamoxifen $(45.6 \%, P=0.0098) .{ }^{18}$ It is important to note that, while these trials were prospectively designed with the idea of a combined analysis, there was considerable patient heterogeneity in terms of hormone receptor status between the trials. Specifically, within TARGET, up to $55 \%$ of participants had unknown hormone receptor status. In comparison, only $11 \%$ of participants in the US trial had unknown hormone receptor status. It is well recognized that tumors expressing hormone receptors derive significant benefit from hormonal therapy; in contrast, hormone receptor-negative tumors are not impacted significantly by the addition of hormonal therapy. Including a number of patients with uncertain hormonal status in these studies may have diluted a differential effect by the hormonal agent.

The combined analysis for the US and TARGET studies suggested that anastrozole $1 \mathrm{mg}$ orally daily was comparable with tamoxifen $20 \mathrm{mg}$ /day orally. ${ }^{20}$ With a median follow-up of 18 months, the median time to disease progression was 
Table I Phase III trials of aromatase inhibitors vs tamoxifen as first-line therapy for metastatic breast cancer

\begin{tabular}{|c|c|c|c|c|c|c|c|c|c|c|c|}
\hline \multirow[t]{2}{*}{ Al agent } & \multirow[t]{2}{*}{$\mathbf{N}$} & \multicolumn{2}{|c|}{ Median TTP } & \multicolumn{2}{|c|}{ Median OS } & \multicolumn{2}{|c|}{ Median TTF } & \multicolumn{2}{|c|}{ Clinical benefit } & \multicolumn{2}{|l|}{ ORR } \\
\hline & & Al & TAM & Al & TAM & Al & TAM & Al & TAM & Al & TAM \\
\hline Anastrozole ${ }^{19}$ & 668 & $8.2 \mathrm{mo}$ & $8.3 \mathrm{mo}$ & NR & NR & $6.2 \mathrm{mo}$ & $6.0 \mathrm{mo}$ & $56.2 \%$ & $55.5 \%$ & $32.9 \%$ & $32.6 \%$ \\
\hline Anastrozole ${ }^{18}$ & 353 & $11.1 \mathrm{mo}$ & $5.6 \mathrm{mo}$ & $N R$ & NR & $7.6 \mathrm{mo}$ & $5.4 \mathrm{mo}$ & $59 \%$ & $46 \%$ & $21 \%$ & $17 \%$ \\
\hline Letrozole $^{21}$ & 907 & $9.4 \mathrm{mo}$ & $6.0 \mathrm{mo}$ & $34 \mathrm{mo}$ & $30 \mathrm{mo}$ & $9.0 \mathrm{mo}$ & $5.7 \mathrm{mo}$ & $50 \%$ & $38 \%$ & $32 \%$ & $21 \%$ \\
\hline Exemestane $e^{22}$ & 371 & NR & NR & $37 \mathrm{mo}$ & $43 \mathrm{mo}$ & $9.9 \mathrm{mo}$ & $5.8 \mathrm{mo}^{*}$ & NR & NR & $46 \%$ & $31 \%$ \\
\hline
\end{tabular}

Note: *Results were reported as progression-free survival in this trial.

Abbreviations: Al, aromatase inhibitor; mo, months; TTP, time to progression; OS, overall survival; TTF, time to failure; ORR, overall response rate; TAM, tamoxifen; NR, not reported.

8.5 months for anastrozole compared with 7 months for the tamoxifen group. In a retrospective subgroup analysis, women with known hormone receptor-positive disease (about $60 \%$ of the total combined study population) had a longer time to disease progression when treated with anastrozole (10.7 months vs 6.4 months) in comparison with tamoxifen-treated patients $(P=0.022)$. In terms of tumor response, the objective response rate was $29 \%$ for anastrozole-treated women and $27.1 \%$ for tamoxifen. The clinical benefit rate also favored anastrozole at $57 \%$ in comparison with $52 \%$ for tamoxifen treatment $(P=0.1129){ }^{20}$

Letrozole has also been compared against tamoxifen in the first-line setting for metastatic breast cancer by the International Letrozole Breast Cancer Group. ${ }^{21}$ This study evaluated 916 patients from November 1996 until January 1999 and elicited more favorable results in patients treated with letrozole. At a median follow-up of 32 months, women treated with letrozole had a 3-month improvement in time to progression when compared with tamoxifen (median 9.4 vs 6.0 months, respectively, $P<0.0001$ ), as well as a better objective response rate ( $32 \%$ vs only $21 \%, P=0.0002$ ) and longer time to treatment failure $(9.0$ months vs 5.7 months, $P<0.0001)$. Women treated with letrozole were also found to have a longer time to chemotherapy, with a median of 16 months, suggesting that it may provide patients with a better quality of life than tamoxifen, which delayed chemotherapy by 9 months $(P=0.005)$. No statistically significant benefit in overall survival could be demonstrated with letrozole treatment, although this was numerically prolonged for the women initially randomized to letrozole. Women on this study were allowed to cross over, and approximately $50 \%$ of women in each arm did transition to the opposite drug, perhaps diluting a survival signal. ${ }^{21}$

Exemestane has also been compared with tamoxifen in postmenopausal women with metastatic breast cancer. ${ }^{22}$ A total of 371 patients were assigned to receive daily oral treatment with either exemestane or tamoxifen. Women treated with exemestane did have an early progression-free survival advantage $(66.2 \%$ vs $49.4 \%$ in tamoxifen-treated women) which diminished over time to $41.7 \%$ in comparison with $31.2 \%$ for tamoxifen-treated women at 12 months. This translated into a statistically insignificant difference in the two progression-free survival curves by the log rank test and Kaplan-Meier analysis. In addition, no advantage was identified. However, tumor response rates were higher for exemestane, with an objective response rate of $46 \%$ compared with $31 \%$ for tamoxifen-treated women, and fewer exemestanetreated women had evidence of disease progression at 29 months of follow-up in comparison with tamoxifen-treated women ( $18.1 \%$ vs $28.6 \%$, respectively). ${ }^{22}$

The optimal sequence of hormonal therapy for women with advanced breast cancer remains ill-defined. However, selective aromatase inhibitors have been identified by the NCCN (National Comprehensive Cancer Network) as preferred first-line therapy for postmenopausal women who have received prior antiestrogen therapy, are within 1 year of antiestrogen exposure, antiestrogen-naïve, or greater than 1 year out from prior antiestrogen therapy. ${ }^{23}$ Women who have had progression of disease on a nonsteroidal aromatase inhibitor may respond to a steroidal aromatase inhibitor and vice versa, although responses in this setting are modest. ${ }^{24-27}$

\section{Adjuvant treatment}

Five years of treatment with the selective estrogen receptor modulator, tamoxifen, for many years considered the gold standard, has been shown to reduce annual breast cancer recurrence rates by almost one half and breast cancer mortality by nearly one-third. ${ }^{6}$ Aromatase inhibitors have now been demonstrated to improve efficacy in numerous large randomized trials compared with tamoxifen in postmenopausal women, given alone in the adjuvant setting as monotherapy for 5 years, sequential therapy sequenced with tamoxifen, or as extended therapy following 5 years of tamoxifen treatment (Table 2). The publication of new data over the past 6 years led the American Society of Clinical Oncology to issue an update to the clinical practice guidelines, which identified 12 major trials using aromatase 
Table 2 Summary of adjuvant trials comparing aromatase inhibitors to tamoxifen for upfront, sequential, and extended use

\begin{tabular}{|c|c|c|c|c|c|c|c|c|c|}
\hline & \multirow[t]{2}{*}{ Trial } & \multirow[t]{2}{*}{ Agent } & \multicolumn{2}{|c|}{ No. of patients } & \multicolumn{2}{|c|}{ DFS/EFS } & \multicolumn{2}{|l|}{ OS } & \multirow{2}{*}{$\begin{array}{l}\text { Median } \\
\text { follow up }\end{array}$} \\
\hline & & & Al/sequential & Control & HR & $P$ & HR & $\boldsymbol{P}$ & \\
\hline Upfront use trials & ATAC $^{30}$ & ANA & 3125 & 3166 & 0.91 & 0.04 & 0.95 & 0.4 & $120 \mathrm{mo}$ \\
\hline (Al vs TAM) & BIG I-98' & LET & 2463 & 2459 & 0.88 & 0.03 & 0.87 & 0.08 & $76 \mathrm{mo}$ \\
\hline Sequential use trials & ARNO $95^{39}$ & ANA & 489 (ITT) & 490 (ITT) & 0.66 & 0.049 & 0.53 & 0.045 & $30.1 \mathrm{mo}$ \\
\hline \multirow[t]{3}{*}{$($ TAM $\rightarrow$ Al vs TAM) } & ABCSG- $8^{37}$ & ANA & I865 (ITT) & I845 (ITT) & 0.82 & 0.038 & $0.78 *$ & 0.032 & $72 \mathrm{mo}$ \\
\hline & ITA $^{38}$ & ANA & 223 & 225 & 0.57 & 0.005 & 0.57 & 0.1 & $64 \mathrm{mo}$ \\
\hline & $\mathrm{IES}^{35}$ & EXE & 2352 & 2372 & 0.76 & 0.0001 & $0.83^{\dagger}$ & 0.05 & $55.7 \mathrm{mo}$ \\
\hline Planned sequential trials & TEAM $^{40}$ & $\mathrm{TAM} \rightarrow \mathrm{EXE}$ & 4868 & 4898 & 0.97 & 0.6 & 1 & $>0.9$ & $60 \mathrm{mo}$ \\
\hline \multirow[t]{2}{*}{$(\mathrm{TAM} \rightarrow \mathrm{Al}$ vs $\mathrm{Al})$} & $\mathrm{BIG} \mid-98^{31}$ & LET $\rightarrow$ TAM & 1540 & 1546 & 0.96 & NS & 0.9 & NS & $71 \mathrm{mo}$ \\
\hline & & TAM $\rightarrow$ LET & 1548 & 1546 & 1.05 & NS & 1.13 & NS & $71 \mathrm{mo}$ \\
\hline Extended use trials & $M A-17^{4 I}$ & LET & 2593 & 2594 & 0.58 & $<0.001$ & - & NS & $30 \mathrm{mo}$ \\
\hline (Al after completing & NSABP-B33 $3^{43}$ & EXE & 783 & 779 & 0.68 & 0.07 & 0.82 & 0.3 & $30 \mathrm{mo}$ \\
\hline
\end{tabular}

Notes: *Crossover censored; ${ }^{\dagger} 22$ estrogen receptor negative patients were sensored.

Abbreviations: DFS, disease free survial; EFS, event free survival; OS, overall survival; Al, aromatase inhibitor; HR, hazard ratio; ATAC, Arimidex, Tamoxifen, Alone or in Combination (trial); ANA, anastrozole; TAM, tamoxifen; BIG, Breast International Group; LET, letrozole; ARNO, Arimidex-Nolvadex (trial); ABCSG, Austrian Breast and Colorectal Cancer Study Group; ITA, Italian Tamoxifen Anastrozole (trial); IES, Intergroup Exemestane Study; EXE, exemestane; TEAM, Tamoxifen Exemestane Adjuvant Multinational (trial); NSABP, National Surgical Adjuvant Breast and Bowel Project; mo, month.

inhibitors in the adjuvant setting. ${ }^{28}$ Despite new data, recent recommendations were not significantly different from prior guidelines recommending an aromatase inhibitor as either primary, sequential, or extended adjuvant treatment to reduce the risk of breast cancer recurrence compared with tamoxifen alone in postmenopausal women. ${ }^{28,29}$ Deciding when to incorporate an aromatase inhibitor and for how long remains less clear. We will review emerging data supportive of multiple potential strategies on which to base a clinical decision.

\section{Upfront adjuvant treatment}

Two large trials have compared an aromatase inhibitor with tamoxifen as initial therapy for early breast cancer. These are the ATAC (Arimidex, Tamoxifen, Alone or in Combination) trial and the Breast International Group (BIG) 1-98 trials. ${ }^{30,31}$ Both have released updated analyses showing an improved relapse-free survival advantage for the aromatase inhibitor as initial adjuvant therapy, and suggest that its use initially may be important to reduce early events in patients at high risk for early recurrence.

The ATAC trial, first reported in 2002, was the first large randomized trial to demonstrate a possible advantage of an aromatase inhibitor over tamoxifen in the adjuvant setting. ${ }^{32}$ This large, double-blind, double-placebo, three-arm trial compared treatment with anastrozole $1 \mathrm{mg}$, tamoxifen $20 \mathrm{mg}$, or both drugs in combination for 5 years in over 9000 women. The combination group was halted after the first analysis because it showed no improvement in efficacy over tamoxifen monotherapy. ${ }^{32}$

In the ATAC trial, $84 \%$ of participants were documented hormone receptor-positive. ${ }^{32}$ An improved disease-free survival was not observed in hormone-receptor negative patients, confirming that only patients with hormone receptor-positive tumors benefit from endocrine therapy.

The most recent analysis, with a median follow-up of 120 months (10 years), continues to confirm a significant benefit of treatment with anastrozole compared with tamoxifen for disease-free survival (hazards ratio [HR]: 0.91, $P=0.04$ ), time to recurrence (HR: $0.84, P=0.001$ ), and time to distant recurrence (HR: $0.87, P=0.03$ ). No survival advantage has yet to be demonstrated. Like tamoxifen, anastrozole does show a carryover benefit after 5 years of treatment, and anastrozole appears to confer additional benefit over tamoxifen for up to 8 years. ${ }^{30}$

The BIG 1-98 trial is a randomized, double-blind, fourarm Phase III trial including roughly 8000 postmenopausal women. ${ }^{31,33}$ Participants were randomized into one of two monotherapy arms comparing 5 years of treatment with letrozole with 5 years of tamoxifen treatment. Two additional arms compare a switching strategy with women receiving 2 years of either tamoxifen or letrozole followed by the alternative drug for the final 3 years. Results of the switching strategy portion of the trial will be discussed later.

Initial results for BIG 1-98 were presented in $2005 .{ }^{33} \mathrm{With}$ a median follow-up of 25.8 months, letrozole demonstrated an improved disease-free survival advantage over tamoxifen (HR: $0.81, P=0.003$ ) at which point the data and safety committee unblinded the monotherapy arms allowing for participants to cross over to letrozole. Despite a $25 \%$ crossover rate, at the 76-month update, letrozole as monotherapy retained a superior disease-free survival (HR: $0.88, P=0.03$ ) and time to recurrence (HR: $0.85, P=0.05$ ) over tamoxifen monotherapy. ${ }^{31}$ As in the ATAC trial, a survival advantage 
has not been demonstrated to date. In both BIG 1-98 and ATAC, the relapse-free survival benefit of aromatase inhibitors can be appreciated early, after just $1-2$ years. This may provide a rationale for upfront usage of these agents over tamoxifen, especially in higher-risk patients. ${ }^{30,31}$

A recent meta-analysis has been conducted by the Early Breast Cancer Trialists' Collaborative Group comparing 5 years of tamoxifen with 5 years of an aromatase inhibitor as adjuvant hormonal therapy for postmenopausal women. ${ }^{34}$ In total, 9856 women with a mean of 5.8 years of follow-up were included. This large analysis confirmed a significant benefit in relapsefree survival, with a $2.9 \%$ absolute gain at 5 years and $3.9 \%$ absolute gain at 8 years which was highly statistically significant $(P<0.00001)$. No breast cancer-specific or overall survival advantage could be discerned in this large meta-analysis. ${ }^{34}$

\section{Sequential adjuvant treatment}

Sequential therapy in any order seems to be equivalent to monotherapy with an aromatase inhibitor, and both strategies are superior to using tamoxifen monotherapy for 5 years as adjuvant therapy. Multiple individual trials and a recent metaanalysis have demonstrated the efficacy of using an aromatase inhibitor following 2-3 years of tamoxifen compared with continuing tamoxifen for 5 years, and updated analyses have even demonstrated small survival advantages. ${ }^{34-39}$ Three trials have shown a survival advantage for switching to an aromatase inhibitor over continuing tamoxifen. These trials are the Intergroup Exemestane Study (IES), ${ }^{35}$ Austrian Breast and Colorectal Cancer Study Group-8 (ABCSG-8), ${ }^{37}$ and Arimidex-Nolvadex 95 (ARNO 95). ${ }^{39}$ The IES trial evaluated switching to exemestane after adjuvant tamoxifen for 2-3 years. At a median follow-up of 55.7 months, the exemestane group showed an absolute disease-free survival benefit of $3.4 \%$ with HR $0.76(P=0.0001)$, and there was a strong trend towards an overall survival benefit with HR of $0.85(P=0.08)$. When women whose tumors were found to be estrogen receptor-negative (122 patients) were censored, the survival advantage became significant (HR: $0.83, P=0.05)$ and the absolute disease-free survival benefit rose to $3.5 \% .^{35}$

The ABCSG-8 and ARNO 95 studies both evaluated 5 years of tamoxifen in comparison with 2 years of tamoxifen followed by a transition to anastrozole for the final 3 years of treatment. ${ }^{37,39}$ These trials were structured similarly with one key difference, ie, women in the ABSCG-8 trial were randomized immediately after primary treatment before beginning tamoxifen, whereas in ARNO 95 women were randomized after completing 2 years of adjuvant tamoxifen.
A combined analysis of these two trials was performed and with 28 months of median follow-up after the completion of the initial 2 years of tamoxifen therapy, the event-free survival was significantly better in the switching arms as compared with tamoxifen (HR: 0.60, $P=0.0009) .{ }^{36}$ Further follow-up of each of these trials has demonstrated an overall survival advantage for the switching arms; for ARNO 95 (HR: 0.53, $P=0.045)^{39}$ and ABCSG-8 (HR: 0.78, $\left.P=0.032\right) .{ }^{37}$

The Italian Tamoxifen Anastrozole (ITA) study has also evaluated a switching strategy in comparison with 5 years of tamoxifen. ${ }^{38}$ As in ARNO 95, postmenopausal women with early-stage hormone receptor-positive breast cancer were randomized after completing 2-3 years of adjuvant tamoxifen to complete a total of 5 years of tamoxifen therapy or to take anastrozole to complete 5 years of adjuvant therapy. At a median follow-up of 64 months, switching to anastrozole yielded a significant event-free survival (HR: $0.57, P=0.005$ ) and relapse-free survival (HR: 0.56, $P=0.01)$. Numerically there were almost $50 \%$ fewer deaths in the switching arm, 12 deaths as compared with 21 in the tamoxifen only arm, but this was not statistically significant $(P=0.1)$ and potentially may be related to the overall small size of the trial (448 women randomized) and few total number of events. ${ }^{38}$

The recent large meta-analysis also evaluated switching strategies (women taking 2-3 years of tamoxifen followed by 2-3 years of an aromatase inhibitor in comparison with a total of 5 years of tamoxifen therapy alone). ${ }^{34}$ This large analysis encompassed 9015 patients at a mean of 3.9 years of follow-up from the time of switching to an aromatase inhibitor. As in the direct comparison of 5 years of an aromatase inhibitor with 5 years of tamoxifen, there was a highly significant $3.1 \%$ absolute advantage at 3 years and $3.6 \%$ absolute advantage at 6 years for relapse-free survival $(P<0.00001)$. In addition, the meta-analysis for the switching cohort demonstrated an incremental advantage for breast cancer-specific survival $(0.7 \%$ gain at 5 years and $1.7 \%$ gain at 8 years, $P=0.02$ ) as well as overall survival $(1.1 \%$ gain at 5 years and $2.2 \%$ gain at 8 years, $P=0.004)$ in comparison with the tamoxifen alone arm. ${ }^{34}$

Based on the available evidence, if a patient is already on tamoxifen for adjuvant therapy, switching to an aromatase inhibitor, either steroidal or nonsteroidal, offers further risk reduction for relapse-free survival and improves overall survival, albeit by a small absolute percentage. The optimal adjuvant hormonal treatment strategy still remains ill-defined.

Is there an advantage to starting tamoxifen with a plan of switching to an aromatase inhibitor after $2-3$ years vs starting 
with an aromatase inhibitor for 5 years of endocrine therapy? This question is being evaluated in two trials, ie, BIG 1-98 and Tamoxifen Exemestane Adjuvant Multinational (TEAM). ${ }^{31,40}$ Both trials directly compare initial use of an aromatase inhibitor with sequential use following tamoxifen and, to date, neither has found any significant difference in efficacy. The two trials randomized women upfront after primary treatment of their invasive breast cancer. In addition, these trials directly compared sequencing strategies to 5 years of an aromatase inhibitor, whereas prior sequencing trials had compared the sequencing arm to 5 years of tamoxifen, the prior gold standard of hormonal therapy.

The TEAM trial enrolled women with hormone receptorpositive, early invasive breast cancer after primary therapy and randomized participants to the steroidal aromatase inhibitor, exemestane, for 5 years or tamoxifen for 2 years followed by exemestane for 3 years. ${ }^{40}$ At 5 years of follow-up, no significant differences were reported between the two arms regarding disease-free survival ( $85 \%$ vs $86 \%$, HR: 0.97 and $P=0.6)$ or overall survival (91\% for both groups).

In the BIG 1-98 trial, two different sequential arms were included. ${ }^{31}$ Participants were randomized to treatment with tamoxifen for 2 years followed by letrozole for 3 years, or letrozole for 2 years followed by tamoxifen for 3 years. The two other arms of BIG 1-98 were previously described and compared 5 years of tamoxifen with 5 years of letrozole. In regards to the two sequential arms, there was no difference in outcomes compared with the letrozole alone arm after 71 months of follow-up. The 5-year disease-free survival was $86.2 \%$ in the tamoxifen followed by the letrozole group, $87.6 \%$ in the letrozole followed by the tamoxifen group, and $87.9 \%$ in the letrozole alone group, all of which were better than $84.6 \%$ in the tamoxifen alone group. In a node-positive subgroup analysis, there were no statistically significant differences between the sequential groups and the letrozole alone group. However, node-positive women treated in the tamoxifen followed by letrozole arm were found to have a nonsignificant risk of early relapse as compared with the letrozole alone arm after 1 year of treatment. ${ }^{31}$ This may suggest upfront usage of an aromatase inhibitor in women with a high risk of early relapse as opposed to sequencing following tamoxifen.

\section{Extended adjuvant treatment}

Two large trials, the National Cancer Institute of Canada Clinical Trials Group MA-17 and National Surgical Adjuvant Breast and Bowel Project B-33 (NSABP B-33), evaluated the use of an aromatase inhibitor after 5 years of adjuvant tamoxifen. ${ }^{41-43}$ Both studies demonstrated an improved disease-free survival with extended adjuvant therapy. The MA-17 trial included over 5000 postmenopausal women who were within 3 months of completing approximately 5 years of tamoxifen. In this double-blind Phase III trial, women were randomized to receive either letrozole or placebo for 5 years. At the first interim analysis (30 months) the disease-free survival and distant disease-free survival were both significantly better in the letrozole group (HR: $0.58, P<0.001$ and HR: $0.60, P=0.002$, respectively). This resulted in the data safety monitoring committee recommending unblinding the treatment allocation. Overall survival did not vary by arm, although was significantly improved for women with nodepositive disease (HR: $0.61, P=0.04) .{ }^{41}$ In addition, at the time of the unblinding, 2383 women who had initially been randomized to placebo and had not suffered a recurrence were offered to be transitioned to letrozole. A total of 1579 opted for transition and 804 elected to forego further treatment. These women were followed prospectively. While the characteristics of these two groups varied, with the women choosing to take letrozole being on average younger, more likely to have had node-positive disease, and to have received chemotherapy, transitioning to letrozole resulted in a $63 \%$ improvement in disease-free survival, a $61 \%$ reduction in the development of distant disease, and a $70 \%$ reduction in the risk of death. All of these findings were statistically significant, suggesting a possible benefit from adjuvant aromatase inhibition following the completion of 5 years of tamoxifen, even after a 2.5 year interruption of therapy. Of note, these results need to be interpreted cautiously because these women were no longer randomized. ${ }^{42}$

The NSABP B-33 trial included just over 1500 women in a similar trial design using the steroidal aromatase inhibitor, exemestane. ${ }^{43}$ When the results of the MA-17 trial became available, the NSABP data monitoring committee recommended stopping accrual for the trial and unblinding the treatment arms, resulting in a $44 \%$ crossover rate to exemestane. Despite this premature closure of the study, at 30 months median follow-up there was a significant improvement in relapse-free survival $(96 \%$ vs $94 \%, P=0.004)$ and a nonsignificant improvement in disease-free survival in favor of exemestane compared with placebo. ${ }^{43}$ Taken together, the results from MA-17 and NSABP B-33 suggest that aromatase inhibition even after 5 years of adjuvant tamoxifen has continued impact on hormonally responsive disease. The natural history of hormonally responsive breast cancer may be quite long. The identification of those patients most likely to benefit from extended adjuvant endocrine therapy remains 
ill-defined. However, data gained from these two trials suggest that perhaps high-risk node-positive patients should be considered for possible extended adjuvant therapy.

\section{Comparing aromatase inhibitors}

Preclinically there are subtle differences in inhibition of estrogens between the third-generation aromatase inhibitors. Treatment with anastrozole decreased estradiol by $84.9 \%$, letrozole by $87.8 \%$, and exemestane by $92.2 \%{ }^{44-46}$ The clinical significance of these differences in estradiol reduction remains unclear. The National Cancer Institute of Canada Clinical Trialists' Group MA-27 trial was the first adjuvant trial to compare two aromatase inhibitors. ${ }^{47}$ Approximately 7500 women were randomized to receive anastrozole vs exemestane for 5 years of adjuvant therapy. Each of the two arms was further randomized to add on celecoxib or placebo, but the celecoxib portion of the study was discontinued when concerns about cardiac risks and nonsteroidal anti-inflammatory agents emerged. At 4.1 years of follow-up, there were no identifiable differences in eventfree survival, overall survival, or compliance rates, suggesting that the two drugs could be used interchangeably. The side effect profiles were slightly different, with exemestane treatment resulting in less hypertriglyceridemia and hypercholesterolemia and lower rates of osteoporosis, although fracture rates were similar. ${ }^{47}$

The Femara vs Anastrozole Clinical Evaluation (FACE) trial is a randomized, open-label Phase III trial comparing 5 years of adjuvant letrozole vs anastrozole in postmenopausal women with hormone receptor-positive, node-positive, highrisk disease. ${ }^{48}$ This trial has completed accrual as of March 2008 , enrolling over 4000 women but has yet to report. The primary endpoint is disease-free survival at 5 years, with secondary endpoints of time to distant recurrence, time to contralateral breast cancer, and safety. The purpose of this trial is to answer the question as to whether or not preclinical data showing greater suppression of estrogen with letrozole in comparison with anastrozole offers a meaningful clinical benefit in women with node-positive, hormone-sensitive breast cancer.

\section{Conclusion for adjuvant treatment}

Each of the three strategies previously discussed, ie, incorporating an aromatase inhibitor as either initial adjuvant therapy, following 2-3 years or tamoxifen, or after 5 years of tamoxifen, has demonstrated efficacy over tamoxifen alone. Survival advantages have not been uniformly demonstrated, and appear to be associated with a crossover strategy. The high crossover rates (up to $25 \%$ in BIG $1-98^{31}$ and up to $66 \%$ in MA-1742) in the large trials or the relatively good prognosis of patients with early hormone receptor-positive breast cancer may have offset a survival advantage. Concerns have been raised that any survival advantage conferred by improved disease-free survival may be negated by toxicities, but to date no significant increase in mortality not related to breast cancer has been clearly identified. It remains unclear whether it is better to use sequential therapy vs an aromatase inhibitor upfront; however, the results so far show that each has merit. What may be optimal for one patient may not prove to be the best strategy for another. The answer likely lies in more personalized decision-making based on each patient's tumor characteristics, health status, and other comorbidities.

\section{Side effects and tolerability}

As outlined above, multiple, large, randomized, clinical trials have demonstrated incremental improvements in breast cancer outcomes in favor of aromatase inhibitors over tamoxifen. Both tamoxifen and aromatase inhibitors affect breast cancer through estrogenic deprivation via different mechanisms. Tamoxifen is a selective estrogen receptor modulator. In the presence of tamoxifen, systemic and local estrogen levels are not affected, instead tamoxifen successfully competes with estrogen at the estrogen receptor interfering with estrogenic orchestrated transcription. In contrast, aromatase inhibition results in lowered systemic estrogen levels and global estrogenic deprivation and thus reduced estrogenic signaling.

In general, aromatase inhibitors have been shown to be relatively safe, with no significantly increased rates of serious adverse events ${ }^{30,35,38,39}$ or increased rates of cancer mortality unrelated to breast cancer ${ }^{30,31,34,38}$ in comparison with tamoxifen. Common side effects of aromatase inhibitors are well described and include menopausal symptoms, hot flashes, masculinization, acne, musculoskeletal complaints (eg, joint stiffness, arthralgias, carpal tunnel, paresthesias), fatigue, dyslipidemia, and increased liver function tests. ${ }^{35,38,39,47}$ Musculoskeletal complaints are among the most common side effects of aromatase inhibitors, with rates as high as $36 \%$, although less than $10 \%$ report severe symptoms..$^{31,39,40,49,50}$

Adverse events with both tamoxifen and aromatase inhibitors have been high. In IES, up to $92.5 \%$ of patients treated with exemestane and $92.6 \%$ of patients on tamoxifen developed an adverse event, although the majority of adverse events were grade I or II. ${ }^{35}$ In BIG-1-98 women randomized to receive letrozole reported more on protocol-specified adverse events in comparison with those randomized to tamoxifen (2912 vs 2554, respectively), but serious or life-threatening 
adverse events were similar (1.7\% in each arm).$^{33}$ Although specific side effect profiles vary for tamoxifen and aromatase inhibitors, quality of life scores have been similar in several studies. ${ }^{35,51,52}$ In a subprotocol of the ATAC trial, quality of life was assessed using the Functional Assessment of Cancer Therapy-Breast and endocrine subscale questionnaires. They found no significant differences in scores between anastrozole or tamoxifen, but there was a lower drug withdrawal rate for anastrozole compared with tamoxifen $(23.3 \%$ vs $29.7 \%) .{ }^{51}$ Withdrawal rates for aromatase inhibitors in some trials have been better ${ }^{32,53}$ or at least not worse than tamoxifen, and compliance rates between aromatase inhibitors appear fairly comparable. ${ }^{47}$ In a longitudinal pharmacy medical claims database study, approximately $25 \%$ of women in a community setting were found to be noncompliant with aromatase inhibitor therapy within the first year of initiation of therapy, and adherence was found to decline over time. ${ }^{54}$ Therefore, the more familiar clinicians are with possible side effects, the earlier intervention may occur and result in improved compliance.

Inhibition of aromatase results in reduced systemic as well as tissue levels of estrogen. Therefore, treatment with aromatase inhibitors predictably avoids many of the proestrogenic gynecological side effects associated with tamoxifen use, such as vaginal bleeding, endometrial hyperplasia, uterine polyps, uterine fibroids ${ }^{35}$ and endometrial cancer. ${ }^{30,31,38,39}$ In contrast, aromatase inhibitors in comparison with tamoxifen are associated with increased vaginal dryness $^{40}$ and reduced libido. ${ }^{53}$

While aromatase inhibitors do avoid some serious adverse effects associated with tamoxifen, such as endometrial hyperplasia/carcinoma and lower rates of thromboembolic events, they are associated with increased rates of bone density loss, osteoporosis development, and fracture risk. In multiple, large, randomized trials, aromatase inhibitors were associated with a statistically significant increased risk of bone fracture while on treatment. ${ }^{30,31,35,36}$ This result was confirmed in a recent meta-analysis, with an absolute $2.2 \%$ increased risk of bone fracture associated with aromatase inhibitors and a number needed to harm of $46 .{ }^{55}$ However, the results of the ATAC and IES studies suggest that aromatase inhibitor-induced adverse effects on bone does not persist once drug therapy is complete..$^{30,56}$

The ARNO 95 study alone demonstrated no significant increase in fracture rates in participants randomized to receive anastrozole following 2 years of tamoxifen in comparison with tamoxifen only. ${ }^{39}$ It has been postulated that tamoxifen pretreatment may have resulted in a bone protective effect. ${ }^{36}$
However, this was not demonstrated in the ABCSG trial 8 or IES studies. ${ }^{35,37}$ In both studies, women received 2-3 years of tamoxifen, and half of the women were then treated with an aromatase inhibitor, anastrozole (ABCSG 8 ) or exemestane (IES), while the remainder continued on tamoxifen. Women in the aromatase inhibitor arm developed significantly more fractures than women treated with tamoxifen alone. Fracture rates in women treated for 5 years with an aromatase inhibitor have been higher than in women treated for 2-3 years with tamoxifen and an aromatase inhibitor. ${ }^{31,40}$ Interestingly, in the BIG 1-98 trial, on switching arms (letrozole followed by tamoxifen vs tamoxifen followed by letrozole) there was actually a lower rate of fracture in the group assigned to letrozole first (7.5\%) rather than tamoxifen first (9.4\%). ${ }^{31} \mathrm{It}$ is premature to draw conclusions as to whether these results can guide any treatment decisions. In a recent observational study, the rate of fractures in patients on aromatase inhibitors was similar to age-matched control patients, whereas the rate of fracture was reduced for patients on tamoxifen as compared with control patients on no endocrine therapy. ${ }^{57}$ These results suggest that aromatase inhibitors may not actually increase the risk of fracture over the general population, but rather that tamoxifen may confer a protective benefit.

The Zoledronic Acid-Letrozole Adjuvant Synergy Trial (Z-FAST) examined whether or not upfront or delayed bisphosphonate administration could mitigate the development of osteoporosis or fracture in postmenopausal women with early breast cancer scheduled to receive an adjuvant aromatase inhibitor. ${ }^{58}$ In this study, postmenopausal women who were to be treated with adjuvant letrozole were randomized to upfront administration of zoledronic acid or delayed initiation of zoledronic acid once the $\mathrm{T}$ score fell below -2.0 or a nontraumatic fracture occurred. Follow-up analyses at months 12, 24, and 36 showed that upfront zoledronic acid administration was more effective than the delayed approach in preventing bone mineral density loss, which is a surrogate marker of fracture risk (absolute difference in mean lumbar spine bone density $6.7 \%, P<0.0001)$. This study was not powered to detect a difference in fracture rates. ${ }^{58}$

Breast cancer patients should be evaluated for risk factors for osteoporosis and have bone mineral density testing at initiation and every 1-2 years while on an aromatase inhibitor. According to the American Society of Clinical Oncologists, all breast cancer patients should receive calcium and vitamin D supplementation and any patient with osteoporosis should receive bisphosphonate therapy. Additionally, any patient with osteopenia should have their treatment individualized based on their risk factors (tools such as the World Health 
Organization absolute fracture risk model, may be used [http://www.sheffield.ac.uk/FRAX/]). ${ }^{59}$ Hadji et al recommend initiating bisphosphonates in women starting aromatase inhibitors who have a $\mathrm{T}$ score less than -2.0 , or two or more risk factors $(\mathrm{T}$ score $<-1.5$, age $>65$ years, body mass index $<20 \mathrm{~kg} / \mathrm{m}^{2}$, family history of hip fracture, personal history of fragility fracture after age 50 years, oral corticosteroid use for more than 6 months, current tobacco use). ${ }^{60}$ Furthermore, bisphosphonate treatment should be considered if an annual bone mineral density decrease of $5 \%$ or more is seen (using the same machine). ${ }^{60}$

Despite gains in relapse-free survival on aromatase inhibitor therapy, the majority of adjuvant trials have been unable to demonstrate a clear superior overall survival advantage in comparison with tamoxifen-treated women. This has raised concerns that perhaps aromatase inhibitors may be associated with side effects which might negatively influence overall survival. The impact of aromatase inhibitors on cardiovascular events, ischemic stroke, and lipid profiles has been monitored closely and several updates have been generated recently.

A review of the individual adjuvant trials shows varying risk for cardiovascular events (excluding thromboembolic events). In one of the smaller switching trials including approximately 1000 women, a higher rate of ischemic cardiovascular events was reported for the anastrozole treatment arm, most of which were angina pectoris. ${ }^{39}$ In the BIG 1-98 trial, an analysis of cardiovascular events conducted with a median follow-up of 30.1 months suggested an overall low rate of events in either treatment arm, but significantly more grade 3-5 events associated with letrozole in comparison with tamoxifen $(P=0.01) .{ }^{61}$ However, after a median follow-up of 71 months, this same trial reported a similar incidence of cardiac events of any grade for any of the letrozolecontaining arms in comparison with tamoxifen $(6.1 \%-7.0 \%$ vs $5.7 \%$, respectively). ${ }^{31}$ In the 10 -year follow-up for the ATAC trial, which was recently reported, there were fewer serious adverse events observed in women treated with anastrozole as compared with tamoxifen (223 vs 369), although this difference resolved in the post-treatment phase. ${ }^{30}$ In addition, no differences in overall mortality, deaths after recurrence, deaths without recurrence, or cardiac deaths were identified. In the TEAM trial, significantly higher rates of arrhythmia, cardiac failure, and hypertension were associated with continuous exemestane treatment as compared with tamoxifen followed by exemestane, but no difference in myocardial infarction rates was observed. ${ }^{40}$ In contrast, in the IES trial, no significant differences were noted by treatment arm for cardiovascular events, although numerically there were more myocardial infarctions in the exemestane-treated group and significantly more hypertension. ${ }^{35}$

Study populations are necessarily unique, and may or may not accurately reflect experiences in the community. In a retrospective review of the Health Core integrated research database, presented at the 2010 San Antonio Breast Cancer symposium, rates of myocardial infarction, ischemic stroke, hip fracture, and all fractures were evaluated in a community population. ${ }^{57}$ Approximately 120,000 women with breast cancer were identified as receiving an aromatase inhibitor, tamoxifen, or no therapy compared with age-matched controls without any identified breast cancer. In this study, the rate of myocardial infarction was not increased in any of the breast cancer groups, regardless of treatment with either tamoxifen or an aromatase inhibitor. The authors concluded that there was not an increased risk of myocardial infarction in the patients treated with an aromatase inhibitor compared with breast cancer patients not on treatment. ${ }^{57}$

Comparing adverse events across trials is somewhat cumbersome because different trials measured different parameters. However, a recent meta-analysis presented by Amir et al at the 2010 San Antonio Breast Cancer Symposium assessed data from the seven main adjuvant aromatase inhibitor trials, with median follow-up of 28-100 months. ${ }^{55}$ This meta-analysis evaluated risk for several potentially life-threatening events, including cardiovascular, cerebrovascular, and thromboembolic events, as well as second cancers, bone fractures, and death without recurrence. For cardiovascular events, there was a $26 \%$ relative increase in events for women treated with an aromatase inhibitor, which was highly statistically significant $(P<0.001)$ and the effect was greatest in women treated with an upfront aromatase inhibitor as compared with those initially treated with tamoxifen and then transitioned to an aromatase inhibitor. While significant, the absolute increase in risk was less than $1 \%(0.8 \%)$ with a number needed to harm of $132 .{ }^{55} \mathrm{~A}$ similar trend was observed in a previously conducted meta-analysis evaluating cardiovascular events associated with the use of third-generation aromatase inhibitors in comparison with tamoxifen. ${ }^{62}$ In this analysis, there was a statistically significant increase in cardiovascular events associated with aromatase inhibitors, although the absolute difference was as small $(0.57 \%, P=0.0038)$, and the number needed to harm was 165 .

In the previously mentioned meta-analysis conducted by Amir et al, no differences in terms of cerebrovascular events, death without recurrence, or second cancers (other than endometrial carcinoma which was associated with tamoxifen usage) were identified. There was a trend towards 
increased death without recurrence in the women treated with upfront aromatase inhibitor therapy as opposed to those who were initially treated with tamoxifen and then switched to an aromatase inhibitor. ${ }^{55}$ This suggests two potential hypotheses; firstly, a possible negative impact associated with duration of aromatase inhibitor treatment and, secondly, a potential mitigating effect of upfront tamoxifen.

Rates of hypercholesterolemia or dyslipidemia have been increased with aromatase inhibitors in most trials, ${ }^{31,38,40}$ but not all. ${ }^{35}$ Impaired lipid metabolism could serve as a mechanism for enhancing cardiovascular disease as a result of treatment with aromatase inhibitors. For instance, in the BIG1-98 trial, hypercholesterolemia rates of any grade overall were lowest for women treated in the tamoxifen alone arm (29.9\%) compared with $41.4 \%-53.2 \%$ in the three letrozole treatment arms. ${ }^{31}$ In the MA-17 trial, evaluation of aromatase inhibitor therapy can be made in comparison with placebo. ${ }^{63}$ However, all women were initially treated with tamoxifen for 5 years which may have potentially imparted a positive impact on cardiovascular health. In this study, after a median follow-up of 30 months, no significant differences were noted for the development of hypertension or hypercholesterolemia. In women over the age of 70 years, rates of cardiac disease were increased, but this did not differ by treatment arm (placebo vs letrozole). ${ }^{63}$ Interestingly, in a single institution report, 52 postmenopausal women to be treated with letrozole were prospectively followed and monitored for total cholesterol, high-density lipoprotein cholesterol, low-density lipoprotein, triglycerides, and estradiol at baseline and at months 3,6 , and 12 of treatment. ${ }^{64}$ In this study, there was a nonsignificant increase in total cholesterol and low-density lipoprotein which reverted back to baseline at the 12-month mark. Triglycerides were elevated and remained elevated throughout the 12-month period, but the increase was considered nonsignificant. Estradiol levels were significantly suppressed at 3 months and remained suppressed throughout. ${ }^{64}$

Thus, the effect of aromatase inhibitors on cardiovascular health remains a contentious issue. The results of the MA-17 trial indicate no significant decrement in the cardiovascular parameters monitored in comparison with placebo. The increased risk in comparison with tamoxifen as suggested by the two meta-analyses may be more a reflection of a mild cardioprotective effect offered by tamoxifen as opposed to a negative impact imparted by aromatase inhibitors. Until these issues are more clearly defined, close monitoring of patients with pre-existing cardiac disease is likely warranted.

\section{Conclusion}

Tamoxifen has been the standard of care for women with hormone receptor-positive breast cancer for the past several decades. Large, randomized, controlled trials conducted in the past two decades have consistently shown an advantage of aromatase inhibitors over tamoxifen for both advanced and early stage breast cancer in postmenopausal women. There continues to be questions regarding the optimal sequencing of hormonal agents. New data are emerging comparing aromatase inhibitors and evaluating their side effect profiles more closely. Choice of hormonal therapy must ultimately be determined based on recurrence risk, individual tolerance, bone health, and the overall side effect profile.

\section{Disclosure}

The authors report no conflicts of interest in this work.

\section{References}

1. Jemal A, Siegel R, Xu J, Ward E. Cancer statistics, 2010. CA Cancer J Clin. 2010;60(5):277-300.

2. Henderson BE, Feigelson HS. Hormonal carcinogenesis. Carcinogenesis. 2000;21(3):427-433.

3. Yager JD. Endogenous estrogens as carcinogens through metabolic activation. J Natl Cancer Inst Monogr. 2000;27:67-73.

4. Cavalieri E, Frenkel K, Liehr JG, Rogan E, Roy D. Estrogens as endogenous genotoxic agents - DNA adducts and mutations. $J$ Natl Cancer Inst Monogr. 2000;27:75-93.

5. Beatson GT. On the treatment of inoperable cases of carcinoma of the mamma: Suggestions for a new method of treatment, with illustrative cases. The Lancet. 1896;2:104-107.

6. Early Breast Cancer Trialists' Collaborative Group. Effects of chemotherapy and hormonal therapy for early breast cancer on recurrence and 15-year survival: an overview of the randomised trials. Lancet. 2005;365(9472):1687-1717.

7. Dowsett M, Haynes BP. Hormonal effects of aromatase inhibitors: Focus on premenopausal effects and interaction with tamoxifen. J Steroid Biochem Mol Biol. 2003;86(3-5):255-263.

8. Casper RF, Mitwally MF. Review: aromatase inhibitors for ovulation induction. J Clin Endocrinol Metab. 2006;91(3):760-771.

9. Nelson LR, Bulun SE. Estrogen production and action. J Am Acad Dermatol. 2001;45(Suppl 3):S116-S124.

10. Simpson ER. Sources of estrogen and their importance. $J$ Steroid Biochem Mol Biol. 2003;86(3-5):225-230.

11. Pasqualini JR, Chetrite G, Blacker C, et al. Concentrations of estrone, estradiol, and estrone sulfate and evaluation of sulfatase and aromatase activities in pre- and postmenopausal breast cancer patients. J Clin Endocrinol Metab. 1996;81(4):1460-1464.

12. Agarwal VR, Bulun SE, Leitch M, Rohrich R, Simpson ER. Use of alternative promoters to express the aromatase cytochrome P450 (CYP19) gene in breast adipose tissues of cancer-free and breast cancer patients. J Clin Endocrinol Metab. 1996;81(11):3843-3849.

13. Chetrite GS, Cortes-Prieto J, Philippe JC, Wright F, Pasqualini JR. Comparison of estrogen concentrations, estrone sulfatase and aromatase activities in normal, and in cancerous, human breast tissues. J Steroid Biochem Mol Biol. 2000;72(1-2):23-27.

14. Lønning PE, Geisler J, Bhatnager A. Development of aromatase inhibitors and their pharmacologic profile. Am J Clin Oncol. 2003;26 (4 Suppl 1):S3-S8.

15. Dombernowsky P, Smith I, Falkson G, et al. Letrozole, a new oral aromatase inhibitor for advanced breast cancer: double-blind randomized trial showing a dose effect and improved efficacy and tolerability compared with megestrol acetate. J Clin Oncol. 1998;16(2):453-461. 
16. Kaufmann M, Bajetta E, Dirix LY, et al. Exemestane is superior to megestrol acetate after tamoxifen failure in postmenopausal women with advanced breast cancer: results of a phase III randomized double-blind trial. The Exemestane Study Group. J Clin Oncol. 2000; 18(7):1399-1411.

17. Buzdar A, Douma J, Davidson N, et al. Phase III, multicenter, doubleblind, randomized study of letrozole, an aromatase inhibitor, for advanced breast cancer versus megestrol acetate. J Clin Oncol. 2001; 19(14):3357-3366.

18. Nabholtz JM, Buzdar A, Pollak M, et al. Anastrozole is superior to tamoxifen as first-line therapy for advanced breast cancer in postmenopausal women: results of a North American multicenter randomized trial. Arimidex Study Group. J Clin Oncol. 2000;18(22):3758-3767.

19. Bonneterre J, Thurlimann B, Robertson JF, et al. Anastrozole versus tamoxifen as first-line therapy for advanced breast cancer in 668 postmenopausal women: Results of the Tamoxifen or Arimidex Randomized Group Efficacy and Tolerability study. J Clin Oncol. 2000;18(22):3748-3757.

20. Bonneterre J, Buzdar A, Nabholtz JM, et al. Anastrozole is superior to tamoxifen as first-line therapy in hormone receptor positive advanced breast carcinoma. Cancer. 2001;92(9):2247-2258.

21. Mouridsen H, Gershanovich M, Sun Y, et al. Phase III study of letrozole versus tamoxifen as first-line therapy of advanced breast cancer in postmenopausal women: Analysis of survival and update of efficacy from the International Letrozole Breast Cancer Group. J Clin Oncol. 2003;21(11):2101-2109.

22. Paridaens RJ, Dirix LY, Beex LV, et al. Phase III study comparing exemestane with tamoxifen as first-line hormonal treatment of metastatic breast cancer in postmenopausal women: The European Organisation for Research and Treatment of Cancer Breast Cancer Cooperative Group. J Clin Oncol. 2008;26(30):4883-4890.

23. National Comprehensive Cancer Network. Version 2.2011 ed. Vol. 2011

24. Harper-Wynne C, Coombes RC. Anastrozole shows evidence of activity in postmenopausal patients who have responded or stabilised on formestane therapy. Eur J Cancer. 1999;35(5):744-746.

25. Chia S, Gradishar W, Mauriac L, et al. Double-blind, randomized placebo controlled trial of fulvestrant compared with exemestane after prior nonsteroidal aromatase inhibitor therapy in postmenopausal women with hormone receptor-positive, advanced breast cancer: results from EFECT. J Clin Oncol. 2008;26(10):1664-1670.

26. Lønning PE, Bajetta E, Murray R, et al. Activity of exemestane in metastatic breast cancer after failure of nonsteroidal aromatase inhibitors: a phase II trial. J Clin Oncol. 2000;18(11):2234-2244.

27. Carlini P, Michelotti A, Ferretti G, et al. Clinical evaluation of the use of exemestane as further hormonal therapy after nonsteroidal aromatase inhibitors in postmenopausal metastatic breast cancer patients. Cancer Invest. 2007;25(2):102-105.

28. Burstein HJ, Prestrud AA, Seidenfeld J, et al. American Society of Clinical Oncology clinical practice guideline: update on adjuvant endocrine therapy for women with hormone receptor-positive breast cancer. J Clin Oncol. 2010;28(23):3784-3796.

29. Winer EP, Hudis C, Burstein HJ, et al. American Society of Clinical Oncology technology assessment on the use of aromatase inhibitors as adjuvant therapy for postmenopausal women with hormone receptor-positive breast cancer: status report 2004. J Clin Oncol. 2005;23(3):619-629.

30. Cuzick J, Sestak I, Baum M, et al. Effect of anastrozole and tamoxifen as adjuvant treatment for early-stage breast cancer: 10-year analysis of the ATAC trial. Lancet Oncol. 2010;11(12):1135-1141.

31. Mouridsen H, Giobbie-Hurder A, Goldhirsch A, et al. Letrozole therapy alone or in sequence with tamoxifen in women with breast cancer. N Engl J Med. 2009;361(8):766-776.

32. Baum M, Budzar AU, Cuzick J, et al. Anastrozole alone or in combination with tamoxifen versus tamoxifen alone for adjuvant treatment of postmenopausal women with early breast cancer: first results of the ATAC randomised trial. Lancet. 2002;359(9324):2131-2139.
33. Thurlimann B, Keshaviah A, Coates AS, et al. A comparison of letrozole and tamoxifen in postmenopausal women with early breast cancer. N Engl J Med. 2005;353(26):2747-2757.

34. Dowsett M, Cuzick J, Ingle J, et al. Meta-analysis of breast cancer outcomes in adjuvant trials of aromatase inhibitors versus tamoxifen. J Clin Oncol. 2010;28(3):509-518.

35. Coombes RC, Kilburn LS, Snowdon CF, et al. Survival and safety of exemestane versus tamoxifen after 2-3 years' tamoxifen treatment (Intergroup Exemestane Study): a randomised controlled trial. Lancet. 2007;369(9561):559-570.

36. Jakesz R, Jonat W, Gnant M, et al. Switching of postmenopausal women with endocrine-responsive early breast cancer to anastrozole after 2 years' adjuvant tamoxifen: Combined results of ABCSG trial 8 and ARNO 95 trial. Lancet. 2005;366(9484):455-462.

37. Jakesz R, Gnant M, Greil R, et al. Tamoxifen and anstrozole as sequencing strategy in post menopausal women with hormone-responsive breast cancer: up date from the Austrian Breast and Colorectal Cancer Study Group Trial 8. Abstract presented at the 31st Annual San Antonio Breast Cancer Symposium, San Antonio, TX, Abstract 14, December 10-14, 2008.

38. Boccardo F, Rubagotti A, Guglielmini P, et al. Switching to anastrozole versus continued tamoxifen treatment of early breast cancer. Updated results of the Italian tamoxifen anastrozole (ITA) trial. Ann Oncol. 2006;17(Suppl 7):vii10-vii14.

39. Kaufmann M, Jonat W, Hilfrich J, et al. Improved overall survival in postmenopausal women with early breast cancer after anastrozole initiated after treatment with tamoxifen compared with continued tamoxifen: The ARNO 95 Study. J Clin Oncol. 2007;25(19):2664-2670.

40. van de Velde CJ, Rea D, Seynaeve C, et al. Adjuvant tamoxifen and exemestane in early breast cancer (TEAM): a randomised phase 3 trial. Lancet. 2011;377(9762):321-331.

41. Goss PE, Ingle JN, Martino S, et al. Randomized trial of letrozole following tamoxifen as extended adjuvant therapy in receptor-positive breast cancer: Updated findings from NCIC CTG MA.17. J Natl Cancer Inst. 2005;97(17):1262-1271.

42. Goss PE, Ingle JN, Pater JL, et al. Late extended adjuvant treatment with letrozole improves outcome in women with early-stage breast cancer who complete 5 years of tamoxifen. J Clin Oncol. 2008; 26(12):1948-1955.

43. Mamounas EP, Jeong JH, Wickerham DL, et al. Benefit from exemestane as extended adjuvant therapy after 5 years of adjuvant tamoxifen: Intention-to-treat analysis of the National Surgical Adjuvant Breast And Bowel Project B-33 trial. J Clin Oncol. 2008;26(12): 1965-1971.

44. Geisler J, Haynes B, Anker G, Dowsett M, Lønning PE. Influence of letrozole and anastrozole on total body aromatization and plasma estrogen levels in postmenopausal breast cancer patients evaluated in a randomized, cross-over study. J Clin Oncol. 2002;20(3): 751-757.

45. Geisler J, King N, Anker G, et al. In vivo inhibition of aromatization by exemestane, a novel irreversible aromatase inhibitor, in postmenopausal breast cancer patients. Clin Cancer Res. 1998;4(9):2089-2093.

46. Geisler J. Differences between the non-steroidal aromatase inhibitors anastrozole and letrozole - of clinical importance? Br J Cancer. 2011;104(7):1059-1066.

47. Goss PE, Ingle JN, Chapman J-AW, et al. Final analysis of NCIC CTG MA.27: A randomized phase III trial of exemestane versus anastrozole in postmenopausal women with hormone receptor positive primary breast cancer. Abstract presented at the 33rd Annual San Antonio Breast Cancer Symposium, San Antonio, TX, Abstract S1-1, December 6-10, 2010.

48. O'Shaughnessy J. A decade of letrozole: FACE. Breast Cancer Res Treat. 2007;105(Suppl 1):67-74.

49. Howell A. Adjuvant aromatase inhibitors for breast cancer. Lancet. 2005;366(9484):431-433

50. Dent SF, Gaspo R, Kissner M, Pritchard KI. Aromatase inhibitor therapy: Toxicities and management strategies in the treatment of postmenopausal women with hormone-sensitive early breast cancer. Breast Cancer Res Treat. 2011;126(2):295-310. 
51. Cella D, Fallowfield L, Barker P, Cuzick J, Locker G, Howell A. Quality of life of postmenopausal women in the ATAC ("Arimidex", tamoxifen, alone or in combination) trial after completion of 5 years' adjuvant treatment for early breast cancer. Breast Cancer Res Treat. 2006;100(3):273-284.

52. Whelan TJ, Goss PE, Ingle JN, et al. Assessment of quality of life in MA.17: a randomized, placebo-controlled trial of letrozole after 5 years of tamoxifen in postmenopausal women. $J$ Clin Oncol. 2005;23(28):6931-6940.

53. Buzdar A, Howell A, Cuzick J, et al. Comprehensive side-effect profile of anastrozole and tamoxifen as adjuvant treatment for early-stage breast cancer: Long-term safety analysis of the ATAC trial. Lancet Oncol. 2006;7(8):633-643.

54. Partridge AH, LaFountain A, Mayer E, Taylor BS, Winer E, AsnisAlibozek A. Adherence to initial adjuvant anastrozole therapy among women with early-stage breast cancer. J Clin Oncol. 2008;26(4): $556-562$.

55. Amir E, Oncaña A, Niraula S, Carlsson L, Seruga B. Toxicity of adjuvant endocrine therapy in postmenopausal breast cancer patients. Abstract presented at the 33rd Annual San Antonio Breast Cancer Symposium, San Antonio, TX, Abstract S2-7, December 6-10, 2010.

56. Coleman RE, Banks LM, Girgis SI, et al. Reversal of skeletal effects of endocrine treatments in the Intergroup Exemestane Study. Breast Cancer Res Treat. 2010;124(1):153-161.

57. Ligbiel JA, O’Malley A, Fisher M, Daniel G, Winer EP, Keating NL. Aromatase inhibitors and risk of myocardial infarction, stroke and fracture. Abstract presented at the 33rd Annual San Antonio Breast Cancer Symposium. San Antonio, TX, Abstract S2-6, December 6-10, 2010.
58. Brufsky AM, Bosserman LD, Caradonna RR, et al. Zoledronic acid effectively prevents aromatase inhibitor-associated bone loss in postmenopausal women with early breast cancer receiving adjuvant letrozole: Z-FAST study 36-month follow-up results. Clin Breast Cancer. 2009;9(2):77-85.

59. Hillner BE, Ingle JN, Chlebowski RT, et al. American Society of Clinical Oncology 2003 update on the role of bisphosphonates and bone health issues in women with breast cancer. J Clin Oncol. 2003;21(21):4042-4057.

60. Hadji P, Body JJ, Aapro MS, et al. Practical guidance for the management of aromatase inhibitor-associated bone loss. Ann Oncol. 2008;19(8):1407-1416.

61. Mouridsen H, Keshaviah A, Coates AS, et al. Cardiovascular adverse events during adjuvant endocrine therapy for early breast cancer using letrozole or tamoxifen: Safety analysis of BIG 1-98 trial. J Clin Oncol. 2007;25(36):5715-5722.

62. Cuppone F, Bria E, Verma S, et al. Do adjuvant aromatase inhibitors increase the cardiovascular risk in postmenopausal women with early breast cancer? Meta-analysis of randomized trials. Cancer. 2008;112(2):260-267.

63. Muss HB, Tu D, Ingle JN, et al. Efficacy, toxicity, and quality of life in older women with early-stage breast cancer treated with letrozole or placebo after 5 years of tamoxifen: NCIC CTG intergroup trial MA.17. J Clin Oncol. 2008;26(12):1956-1964.

64. Zidan J, Chetver L, Hussein O, Zucker M. Effect of letrozole on plasma lipids, triglycerides, and estradiol in postmenopausal women with metastatic breast cancer. Oncologist. 2010;15(11):1159-1163.
Breast Cancer: Targets and Therapy

\section{Publish your work in this journal}

Breast Cancer: Targets and Therapy is an international, peerreviewed open access journal focusing on breast cancer research, identification of therapeutic targets and the optimal use of preventative and integrated treatment interventions to achieve improved outcomes, enhanced survival and quality of life for the cancer patient.

\section{Dovepress}

View the full aims and scopes of this journal here. The manuscript management system is completely online and includes a very quick and fair peer-review system, which is all easy to use. Visit http:// www.dovepress.com/testimonials.php to read real quotes from published authors. 